\title{
Increased plasma microRNA and CD133/CK18-positive cancer cells in the pleural fluid of a pancreatic cancer patient with liver and pleural metastases and correlation with chemoresistance
}

\author{
CHUANLI REN, ${ }^{1}$ HUI CHEN ${ }^{2}$, CHONGXU HAN $^{1}$, DAXIN WANG $^{3}$ and DEYUAN FU ${ }^{4}$ \\ ${ }^{1}$ Medical Laboratory; ${ }^{2}$ Geriatric Medicine Department; ${ }^{3}$ Research Centre of Biomedical Engineering; \\ ${ }^{4}$ General Surgery Department, Northern Jiangsu People's Hospital and Clinical Medical College of Yangzhou University, \\ Yangzhou 225001, P.R China
}

Received March 26, 2012; Accepted July 4, 2012

DOI: $10.3892 / \mathrm{ol} .2012 .798$

\begin{abstract}
We report a case of notably increased plasma levels of microRNA (miR)-21, miR-25, miR-103 and miR-151 in a pancreatic cancer patient with liver and pleural metastases. CD45-coated immunomagnetic beads detected an enrichment of malignant cancer cells in the pleural fluid, and $\mathrm{CD} 133^{+} \mathrm{CK} 18^{+}$cancer cells were identified. Using computer tomography (CT) combined with cancer cells stained in the pleural fluid, a previously healthy 60-year-old male was diagnosed with pancreatic cancer with multiple liver tumor metastases. Cancer antigen 19-9 (CA19-9), alkaline phosphatase (ALP) and $\gamma$-glutamate-transpeptidase $(\gamma$-GT) were notably increased in the serum, and carcinoembryonic antigen (CEA) was increased in the pleural fluid. The patient succumbed to the disease three months following standard chemotherapy. The increased levels of plasma miR-21, miR-25, miR-103 and miR-151, as well as the identification of CD133 ${ }^{+} \mathrm{CK} 18^{+}$cells in the pleural fluid of a pancreatic cancer patient with liver metastases, may regulate the molecular mechanisms involved in chemoresistance. The patient was insensitive to chemotherapy and succumbed 3 months later. Full elucidation of the molecular and pathological features of pancreatic cancer may be a novel strategy for diagnosis and tailored therapy.
\end{abstract}

\section{Introduction}

Pancreatic cancer is a highly aggressive malignancy with extremely poor prognosis that is considered to be partly

Correspondence to: Dr Chuanli Ren or Dr Chongxu Han, Medical Laboratory, Northern Jiangsu People's Hospital and Clinical Medical College of Yangzhou University, No. 17 Nantong West Road, Yangzhou 225001, P.R. China

E-mail: renchl@163.com

E-mail: hanchongxu66@163.com

Key words: pancreatic cancer, liver metastases, CD133, plasma microRNA, pleural fluid, tailored therapy due to the chemotherapy-resistant characteristics of specific pancreatic cancer cell subgroups. CD133 and CXCR4 are potential markers of pancreatic cancer stem cells (CSCs), which form tumor tissues and promote tumor progression and metastasis (1). It has been suggested that microRNAs (miRs) are critical regulators of tumor progression and drug resistance in pancreatic cancer cells (2). Low miR-21 expression in tumor tissues has been associated with beneficial adjuvant treatment in pancreatic cancer cases, and it has been revealed that anti-miR-21 increases anticancer drug activity in vitro (3).

We used the methods described in our previous study (4) to enrich rare cells from the pleural fluid and to analyze the expression of the CSC marker CD133, and the epithelial marker, cytokeratin 18 (CK18). The plasma levels of miR-21, miR-25, miR-103, miR-151 and cancer antigen 19-9 (CA19-9) in the serum, and the clinical pathological parameters of the patients, were also studied. Following therapy, the condition of the patient was monitored until mortality.

\section{Materials and methods}

Enrichment of cancer cells from pleural effusion. The patient, who provided informed consent, was enrolled using institutional review board-approved protocols. Pleural effusion $(10 \mathrm{ml})$ was collected from the patient into acid citrate dextrose venous collection tubes (Becton Dickinson, Franklin Lakes, NJ, USA) and transferred into a 50-ml centrifuge tube. Malignant cancer cells were enriched from the pleural fluid using CD45-coated immunomagnetic beads (Cyttel Biosciences, Beijing, China) following the method described in our previous study (4). The cell pellet was then transferred onto glass slides for further analysis.

Immunofluorescence (IF) staining. Double IF staining was conducted using $100 \mu \mathrm{l}$ anti-CK18-FITC (green) and anti-CD133-PE (red; Miltenyi Biotec, Bergisch Gladbach, Germany). The cell pellet was transferred onto glass slides and incubated with the labeled primary antibodies (1:100 dilution in $2 \% \mathrm{BSA}$ ) for $60 \mathrm{~min}$ at room temperature. The nuclei were counterstained with DAPI and a blinded review of three-color 
Table I. Laboratory data of pleural effusion from the pancreatic cancer patient with liver and pleural metastasis.

\begin{tabular}{lccc}
\hline Variable & Control & Result & Clinical significance \\
\hline Color & Colorless & Yellow & Abnormal \\
Trait & Clear & Muddy & Abnormal \\
Pleural chylous test & Negative & Negative & Normal \\
Acid-fast staining & Negative & Negative & Normal \\
Cell counting (number/l) & Negative & $0.5 \times 10^{9}$ & Normal \\
Total protein $(\mathrm{g} / \mathrm{dl})$ & $6.0-8.0$ & 4.4 & Decreased \\
Lactate dehydrogenase $(\mathrm{U} / \mathrm{l})$ & $106-246$ & 211 & Normal \\
Adenosine deaminase $(\mathrm{U} / \mathrm{l})$ & $0-25$ & 13 & Normal \\
CEA $(\mathrm{U} / \mathrm{l})$ & $<5$ & 103 & Tumor metastasis \\
Cancer cell staining $(/ \mathrm{ml})$ & Negative & 30 & Malignant diseases diagnosis \\
CD133 ${ }^{+} \mathrm{CK} 18^{-}$cells & Negative & Positive & Maybe CSCs \\
$\mathrm{CD} 133^{+} \mathrm{CK} 18^{+}$cells & Negative & Positive & Maybe CSCs \\
CK18 ${ }^{+} \mathrm{CD} 133^{-}$cells & Negative & Positive & Malignant epithelial cells \\
\hline
\end{tabular}

CEA, carcinoembryonic antigen; CSCs, cancer stem cells.

fluorescence images by three examiners (magnification, $\mathrm{x} 400$ ) confirmed the identity of $\mathrm{CD} 133^{+} \mathrm{CK} 18^{+}$cells.

Plasma RNA isolation. Total RNA from the plasma of the pancreatic cancer patient and from the age- and gender-matched healthy donors was isolated using TRIzol for RNA (Invitrogen, Carlsbad, CA, USA) according to the manufacturer's instructions. Circulating miRNA was isolated using a mirVana PARIS kit (Applied Biosystems, Foster City, CA, USA) according to the manufacturer's instructions. RNA concentration was determined using a NanoDrop ND-1000 spectrophotometer (Thermo Scientific, Worcester, MA, USA) on a denaturing $15 \%$ polyacrylamide gel.

Quantitative reverse transcriptase polymerase chain reaction $(q R T-P C R)$. miR-16, miR-21, miR-103 and miR-151 were quantified in triplicate by qRT-PCR using TaqMan MicroRNA Assay kits (Applied Biosystems). A total of $2.5 \mu 1$ synthetic C. elegans miRNA, cel-miR-39 $\left(2 \times 10^{-3} \mathrm{pmol} / \mu\right.$ l synthetic RNA oligonucleotides; Qiagen, Hilden, Germany), was applied to each sample as an internal control. The method used for the identification of miR-16, miR-21, miR-103 and miR-151 in the plasma was described previously (5). The relative abundance of the miRNAs was determined using the following equation: Relative miRNA abundance $=-\Delta \Delta \mathrm{Ct}=-\left[\left(\right.\right.$ Sample $\mathrm{Ct}_{\text {target }}-$ Sample $\left.\mathrm{Ct}_{\text {cell-miR-39 }}\right)-$ (Control $\mathrm{Ct}_{\text {target }}-$ Control $\left.\mathrm{Ct}_{\text {cell-miR-39) }}\right)$.

\section{Case report}

A 60 year-old male was admitted to the Northern Jiangsu People's Hospital and Clinical Medical College of Yangzhou University (Yangzhou, China) having suffered symptoms of abdominal distention and anorexia for one month. The patient had been healthy and had no history of malignant or other common diseases. The patient did not smoke, but had been addicted to alcohol for 20 years with a $250 \mathrm{~g}$ average daily intake of alcohol. Serum alkaline phosphatase (ALP),
A

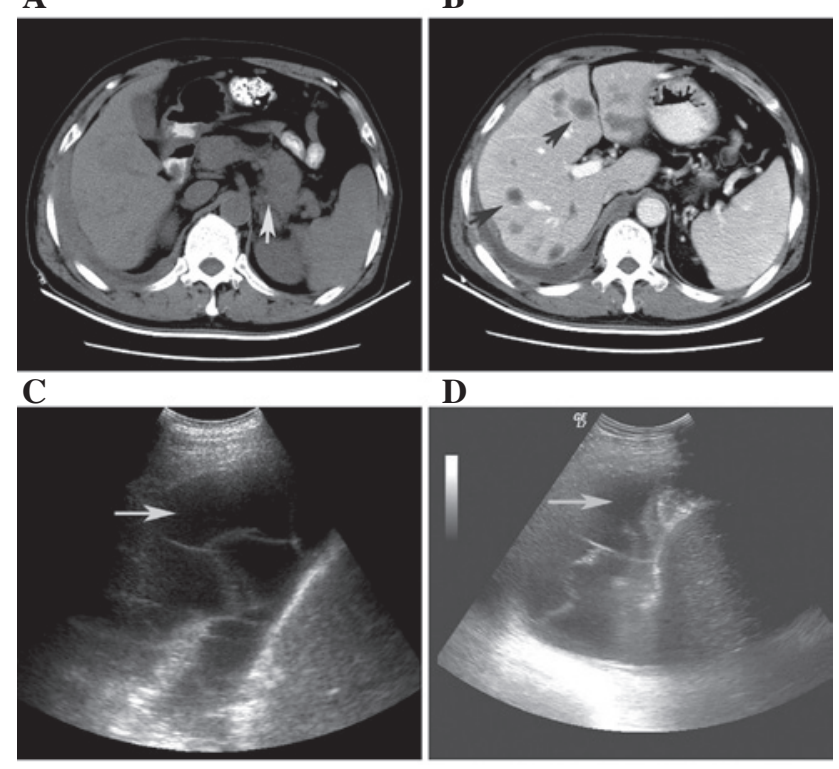

Figure 1. (A) CT scan of the primary tumor in the pancreas. An axial image of the abdomen identified a mass in the body-tail of the pancreas of $43 \times 48 \mathrm{~mm}$ (arrow). (B) CT detection of multiple tumor metastases in the liver (arrows). (C and D) Abdominal ultrasonographic detection of pleural fluid in the right chest. CT, computer tomography.

$\gamma$-glutamate-transpeptidase $(\gamma$-GT) and CA19-9 levels were $195 \mathrm{U} / \mathrm{l}, 291 \mathrm{U} / \mathrm{l}$ and $1200 \mathrm{U} / \mathrm{ml}$, respectively; all of which are significantly elevated compared with normal levels.

Abdominal ultrasonographic diagnosis indicated pleural effusion in the right chest (Fig. 1C and D). The features of the pleural fluid are shown in Table I. A total of 50 malignant cancer cells $/ \mathrm{ml}$ were identified in the pleural fluid, while carcinoembryonic antigen (CEA) was elevated to $103.9 \mathrm{U} / 1$. The $43 \times 48 \mathrm{~mm}$ mass in the body-tail of the pancreas was detected using computer tomography (CT; Fig. 1A). A different nodus size was detected at low density in the liver. The maximum 
Table II. Circulating miRNA-21, miRNA-25, miRNA-103 and miRNA-151 levels in the pancreatic cancer patient and in five age- and gender-matched healthy donors.

\begin{tabular}{|c|c|c|c|c|}
\hline Sample & miR-21 & miR-25 & miR-103 & $\operatorname{miR}-151$ \\
\hline Pancreatic cancer $(\mathrm{n}=1)(\mathrm{pmol} / \mu \mathrm{l})$ & $2.5 \times 10^{-4}$ & $0.24 \times 10^{-4}$ & $0.34 \times 10^{-4}$ & $0.71 \times 10^{-4}$ \\
\hline Healthy control $(\mathrm{n}=5)(\mathrm{pmol} / \mu \mathrm{l})$ & $0.3 \times 10^{-4}$ & $0.12 \times 10^{-4}$ & $0.05 \times 10^{-4}$ & $0.16 \times 10^{-4}$ \\
\hline $\begin{array}{l}\text { Multiple of increase compared } \\
\text { with healthy control }\end{array}$ & 8.3 & 2.0 & 6.8 & 4.4 \\
\hline
\end{tabular}

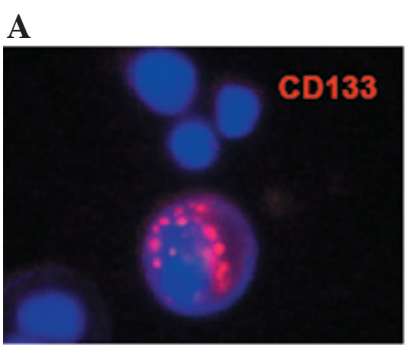

B


C

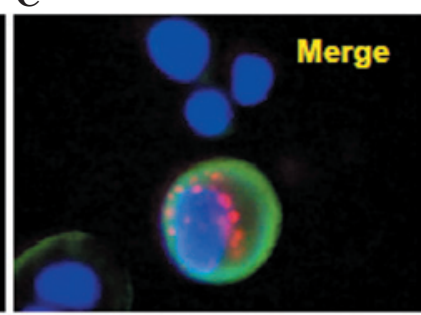

F

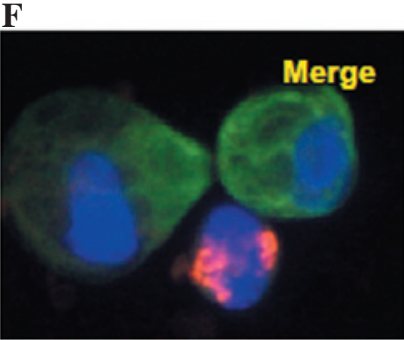

Figure 2. Detection of rare $\mathrm{CD} 133^{+} \mathrm{CK} 18^{+}, \mathrm{CD} 133^{+} \mathrm{CK} 18^{-}$and $\mathrm{CK} 18^{+} \mathrm{CD} 133^{-}$cells in pleural effusion enriched by anti-CD45 antibody coated beads using

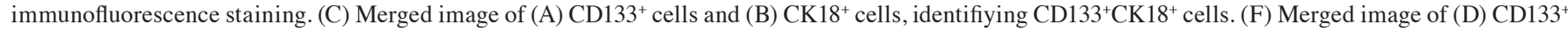
cells and (E) $\mathrm{CK} 18^{+}$cells, identifying cells singly stained for $\mathrm{CD} 133^{+} \mathrm{CK} 18^{-}$or $\mathrm{CK} 18^{+} \mathrm{CD} 133^{-}$.

diameter of the metastatic tumor was $\sim 20 \mathrm{~mm}$ (Fig. 1B). The patient was administered chemotherapy with one cycle of gemcitabine and oxaliplatin combined with abdominal cavity perfusion and gemcitabine. However, the patient was insensitive to the systemic therapy and succumbed to liver metastasis and other complications 3 months later.

The plasma miR-21, miR-25, miR-103 and miR-151 levels were notably increased in the serum of this patient, being 8.3, 2.0, 6.8 and 4.4-times higher compared with that of the average from five age- and gender-matched healthy controls, respectively (Table II). Moreover, malignant cancer cells in the pleural fluid were enriched by CD45-coated immunomagnetic beads. The number of cancer cells in the pleural fluid was enumerated as $30 / \mathrm{ml}$ using Wright-Giemsa stain. $\mathrm{CD}_{133}{ }^{+} \mathrm{CK} 18^{+}, \mathrm{CD} 133^{+} \mathrm{CK} 18^{-}$and $\mathrm{CK} 18^{+} \mathrm{CD} 133^{-}$cancer cells were detected in the pleural fluid of the patient (Table I; Fig. 2).

\section{Discussion}

Although the only potentially curative treatment for pancreatic ductal adenocarcinoma is surgical resection, the vast majority of patients are diagnosed at stages that are too advanced to undergo curative surgery. Therefore, biomarkers for early detection and new therapeutic strategies are urgently required.
Pancreatic cancer has unique miRNA expression patterns, which are different from those of other cancers, and enable the differentiation of normal pancreatic tissue from benign inflammatory pancreatic tissue (6). Overexpression of miR-21 in tumors is markedly associated with liver metastasis of pancreatic cancer (7). In this patient, the plasma levels of miR-21 were 8.3 times higher than those of the age- and gender-matched healthy controls. The levels of the other three miRNAs examined, miR-25, miR-103 and miR-151, were also 2.0, 6.8 and 4.4 times higher compared with those of the healthy controls, respectively (Table II). In this study, multiple liver metastases and pleural fluid in the right chest were detected by CT and ultrasonographic examination (Fig. 1). Furthermore, increased serum CA19-9, ALP and $\gamma$-GT levels indicated malignant disease and liver metastasis. As this patient was not fit for surgery, the diagnosis of pancreatic cancer by pathology was not acquired. However, the diagnosis could be confirmed by clinical symptoms, imaging diagnosis and laboratory examinations.

We employed a method using CD45-coated immunomagnetic beads to enrich cancer cells from pleural blood $(4,8)$. A total of 30 cancer cells/ml pleural effusion were enriched and detected (Table I). $\mathrm{CD} 133^{+} \mathrm{CK} 18^{+}$double positive cells as well as $\mathrm{CD} 133^{+} \mathrm{CK} 18^{-}$and $\mathrm{CK} 18^{+} \mathrm{CD} 133^{-}$single positive cells, were detected in the pleural fluid of this patient. Our results 
support the recent findings from the Stanger research group, which identified that circulating pancreatic cells maintained a mesenchymal phenotype, exhibited stem cell properties and seeded the liver in animal models of pancreatic cancer (9).

As CKs are markers of epithelial cells or epithelial originated tumors, they are often used to judge whether cells in serous effusions are derived from epithelial cells (10). The detection of $\mathrm{CK} 18^{+}$cells in the pleural fluid implied that these cells may have originated from epithelial tumor cells. The different states of $\mathrm{CD}_{133^{+}}$cancer cells in the pleural fluid may reflect the heterogeneity of these malignant cancer cells. It has been revealed that CD133 expression in pancreatic cancer was exclusively tumorigenic and highly resistant to standard gemcitabine chemotherapy $(1,11)$. Cyclopamine is an effective method of reversing gemcitabine resistance in pancreatic cancer with high levels of cancer stem cell markers (11). A small number of cancers have CD133-expressing cells in pleural effusion, which may imply resistance to standard chemotherapy. Since the patient was admitted to hospital with multiple liver metastasis and pleural effusion, alleviative chemotherapy with gemcitabine and oxaliplatin combined with abdominal cavity perfusion chemotherapy and gemcitabine was administered. However, the patient was insensitive to the chemotherapy and succumbed to liver metastasis and other complications three months later. The patient may have benefited from treatment with cyclopamine or other targeted therapies. The most highly increased miRNA level in the plasma of this patient was that of miR-21. Downregulation of miR-21 results in an increased sensitivity of these cells to gemcitabine $(3,12-14)$. Measurement of plasma miR-21 levels and serum CA19-9 may be helpful for early detection of pancreatic cancer (5).

To the best of our knowledge, this is the first study of $\mathrm{CD}_{133^{+}}$cancer cells in the pleural effusion of a pancreatic cancer patient with liver metastasis. Detection of CD133 ${ }^{+}$ cancer cells in the pleural effusion of this patient may imply the chemoresistance and cancer stem feature of these cells, which may provide physicians with significant information for tailored treatment and evaluating prognosis. In addition, the plasma miR-21, miR-25, miR-103 and miR-151 levels were also increased compared with the healthy controls. This molecular feature of the patient is consistent with the clinical pathological parameters. Full elucidation of the molecular and pathological features of pancreatic cancer may be a novel strategy for tailored therapy.

\section{Acknowledgements}

This study was supported by the National Natural Science Foundation of China (No. 81172508), the Foundation of the Health Department Program of Jiangsu Province (No. 200970) and the Foundation of the Social Development of Jiangsu Province (No. SBE201270298). We thank Dr Pin Lin and Dr Xingxiang $\mathrm{Xu}$ for excellent experimental assistance.

\section{References}

1. Hermann PC, Huber SL, Herrler T, et al: Distinct populations of cancer stem cells determine tumor growth and metastatic activity in human pancreatic cancer. Cell Stem Cell 1: 313-323, 2007.

2. Wang Z, Li Y, Ahmad A, et al: Pancreatic cancer: understanding and overcoming chemoresistance. Nat Rev Gastroenterol Hepatol 8: 27-33, 2011.

3. Stokes JB, Adair SJ, Slack-Davis JK, et al: Inhibition of focal adhesion kinase by PF-562,271 inhibits the growth and metastasis of pancreatic cancer concomitant with altering the tumor microenvironment. Mol Cancer Ther 10: 2135-2145, 2011.

4. Ren C, Han C, Zhang J, et al: Detection of apoptotic circulating tumor cells in advanced pancreatic cancer following 5-fluorouracil chemotherapy. Cancer Biol Ther 12: 700-706, 2011.

5. Liu J, Gao J, Du Y, et al: Combination of plasma microRNAs with serum CA19-9 for early detection of pancreatic cancer. Int J Cancer 131: 683-691, 2011.

6. Park JY, Helm J, Coppola D, Kim D, Malafa M and Kim SJ: MicroRNAs in pancreatic ductal adenocarcinoma. World J Gastroenterol 17: 817-827, 2011.

7. Roldo C, Missiaglia E, Hagan JP, et al: MicroRNA expression abnormalities in pancreatic endocrine and acinar tumors are associated with distinctive pathologic features and clinical behavior. J Clin Oncol 24: 4677-4684, 2006.

8. Ren C, He P, Zhang J, Zheng Z, Qian Y and Zhao X: Malignant characteristics of circulating tumor cells and corresponding primary tumor in a patient with esophageal squamous cell carcinoma before and after surgery. Cancer Biol Ther 11: 633-638, 2011.

9. Rhim AD, Mirek ET, Aiello NM, et al: EMT and dissemination precede pancreatic tumor formation. Cell 148: 349-361, 2012

10. Ascoli V, Taccogna S, Scalzo CC and Nardi F: Utility of cytokeratin 20 in identifying the origin of metastatic carcinomas in effusions. Diagn Cytopathol 12: 303-308, 1995.

11. Yao J, An Y, Wie JS, et al: Cyclopamine reverts acquired chemoresistance and down-regulates cancer stem cell markers in pancreatic cancer cell lines. Swiss Med Wkly 141: w13208, 2011.

12. Giovannetti E, Funel N, Peters GJ, et al: MicroRNA-21 in pancreatic cancer: correlation with clinical outcome and pharmacologic aspects underlying its role in the modulation of gemcitabine activity. Cancer Res 70: 4528-4538, 2010.

13. Ali S, Ahmad A, Banerjee S, et al: Gemcitabine sensitivity can be induced in pancreatic cancer cells through modulation of miR-200 and miR-21 expression by curcumin or its analogue CDF. Cancer Res 70: 3606-3617, 2010

14. Moriyama T, Ohuchida K, Mizumoto K, et al: MicroRNA-21 modulates biological functions of pancreatic cancer cells including their proliferation, invasion, and chemoresistance. Mol Cancer Ther 8: 1067-1074, 2009. 